DOI: $10.20472 /$ IAC.2019.051.023

EVA MALOVICS

University of Szeged, Hungary

BEATA VAJDA

University of Szeged, Hungary

\title{
THE ANALYSIS OF DOCTOR-PATIENT COMMUNICATIONS - AN APPLICATION OF THE GROUNDED THEORY METHOD
}

\begin{abstract}
:
Scientific research on doctor-patient communication dates back for decades, and the current situation of its practical realization has been criticized in many studies at both nation and international level and is generally considered inefficient. Doctor-patient communication, embedded within the framework of the complex health care system is itself a complex subject. According to experts, in a networked society, the digitalization of information and communication changes the way people communicate and cooperate and predict significant changes in communication. The knowledge asymmetry between doctors and patients has decreased with the use of the Internet. Accordingly, our research question is how this phenomenon appears in doctor-patient communication in a Hungarian setting.

In accordance with the complexity of the topic, we chose to use in-depth interviews and focus group interviews as a research method and analyzed them using the Grounded Theory method. Two focus groups were conducted in which both actors, doctors and patients appeared, and we conducted in-depth interviews with four doctors and ten patients. Subjects, in accordance with the Grounded Theory method, were selected by quality sampling. We had an interview guideline, but we deviated from it from time to time, which is allowed by our chosen research method.

The characteristics of communication were interpreted according to the social psychological and functional model. According to our results, the traditional communication culture of healthcare is dominant in communication, doctors adhere to this, which today leads to a decrease in patient trust, which is attempted to be managed by patients with different strategies.
\end{abstract}

\section{Keywords:}

healthcare, communication, grounded theory

JEL Classification: I12 